\title{
Search for CSS and GPS Candidates from the Peacock and Wall Catalogue
}

\author{
A. Rossetti ${ }^{1}$, F. Mantovani ${ }^{1}$, C. Fanti ${ }^{1,2}$ and R. Fanti ${ }^{1,2}$ \\ ${ }^{1}$ Istituto di Radioastronomia del CNR, via Gobetti 101, I-40129 Bologna, Italy \\ rossetti@ira.cnr.it \\ fmantovani@ira.cnr.it \\ ${ }^{2}$ Dipartimento di Fisica, Università degli Studi, via Irnerio 46, I-40126 Bologna, Italy \\ cfanti@ira.cnr.it \\ rfanti@ira.cnr.it
}

Received 2002 June 26, accepted 2002 October 11

\begin{abstract}
Samples of CSS/GPS sources suitable for statistical studies have been selected from the 3CR catalogue and from the Peacock \& Wall catalogue. The selection criterion used for the Peacock \& Wall catalogue CSS/GPS sources selects sources which peak below about $2 \mathrm{GHz}$ and are therefore larger than $\sim 200 \mathrm{pc}$. Our main aim is to find among the flat spectrum sources in the Peacock \& Wall catalogue smaller size CSS/GPS sources by means of systematic high resolution observations. The discovery of a few more would be important in order to finally have a complete flux limited sample of this class of objects. We discuss the results obtained so far for three of the observed sources.
\end{abstract}

Keywords: galaxies: active - quasars: individual $(0153+744,0133+476,0202+149)-$ radio continuum: general

\section{Project Outline}

Samples of CSS/GPS sources suitable for statistical studies have been selected from the 3CR catalogue (Laing, Riley, \& Longair 1983) and from the Peacock \& Wall (1982, hereafter PW) catalogue. The PW catalogue, owing to the higher frequency selection $(2.7 \mathrm{GHz}$ and $5 \mathrm{GHz}$ ), contains a number of GPS sources whose radio spectra turn over at frequencies higher than those of the 3CR, so that it is particularly suitable for searching for small size objects. However, the selection criterion used for PW CSS/GPS selects sources which peak below about $2 \mathrm{GHz}$ and therefore are larger than $\sim 200$ pc (O'Dea 1998). Smaller size sources with turnover frequency between 2 and $5 \mathrm{GHz}$ would appear as flat spectrum sources and therefore would be confused with the class of core-jet objects. Objects like these can be found only by means of systematic high resolution and/or high frequency observations of all the flat spectrum sources in the PW catalogue. Our main aim is to find among them small size CSS/GPS sources for which structure information was not available.

\section{Radio Observations}

Our sample consists of eight sources for which the mas structure is unknown or uncertain. The flux density measurements at high frequencies show that the spectrum for the majority of them is not flat. From the optical identification all objects are QSOs. Six sources have been observed with the VLBA at one or more of the following frequencies: $1.6 \mathrm{GHz}, 5 \mathrm{GHz}, 8.4 \mathrm{GHz}$, and $15 \mathrm{GHz}$. Here we discuss the data for three objects (listed in Table 1) examinated so far.
0153+744. The radio emission at mas scales for this quasar at centimetre wavelengths is dominated by two components (Figure 1). Between the two components there is a 'bridge' of emission (components C, D, E as in Hummel et al. 1997). Parameters for each component are listed in Table 2. We find a spectral index $\alpha=0.2$ $\left(S \propto v^{-\alpha}\right)$ for the North component, as a whole, between 5 and $15 \mathrm{GHz}$ (Table 2). Comparing our results with data from Hummel et al. (1997) we obtain the same value for $\alpha$ between 15 and $22 \mathrm{GHz}$. Component $\mathrm{A}$ is resolved at $22 \mathrm{GHz}$ into a core-jet structure with four distinct components, the second of which, with a flat spectrum, is likely to be the real core. Component $\mathrm{B}$ shows complex structure consisting of resolved steep-spectrum emission. The radio spectrum peaks at around $1 \mathrm{GHz}$ so we can classify this object as a GPS source as proposed by O'Dea, Baum, \& Stanghellini (1991).

0133+476. This source is only slightly resolved by our observations (Figure 2). From the PW catalogue this

Table 1. General source parameters from Johnston et al. (1995)

\begin{tabular}{llccccc}
\hline Name & Type & $z$ & $\mathrm{~m}_{v}$ & $\begin{array}{c}S_{5 \mathrm{GHz}} \\
\mathrm{mJy}\end{array}$ & $\begin{array}{c}S_{8.4 \mathrm{GHz}} \\
\mathrm{mJy}\end{array}$ & $\begin{array}{c}\mathrm{LS} \\
\mathrm{pc}\end{array}$ \\
\hline $0153+744$ & QSO & 2.338 & 16.0 & 1500 & $910^{(1)}$ & $\sim 40$ \\
$0133+476$ & QSO & 0.859 & 19.0 & 3300 & $1699^{(1)}$ & $\sim 54$ \\
$0202+149$ & QSO & $0.405^{(2)}$ & 21.9 & 2400 & $2270^{(3)}$ & $\sim 24$
\end{tabular}

\footnotetext{
(1) Patnaik et al. (1992).

(2) Perlman et al. (1998).

(3) Wright \& Otrupcek (1990).
} 

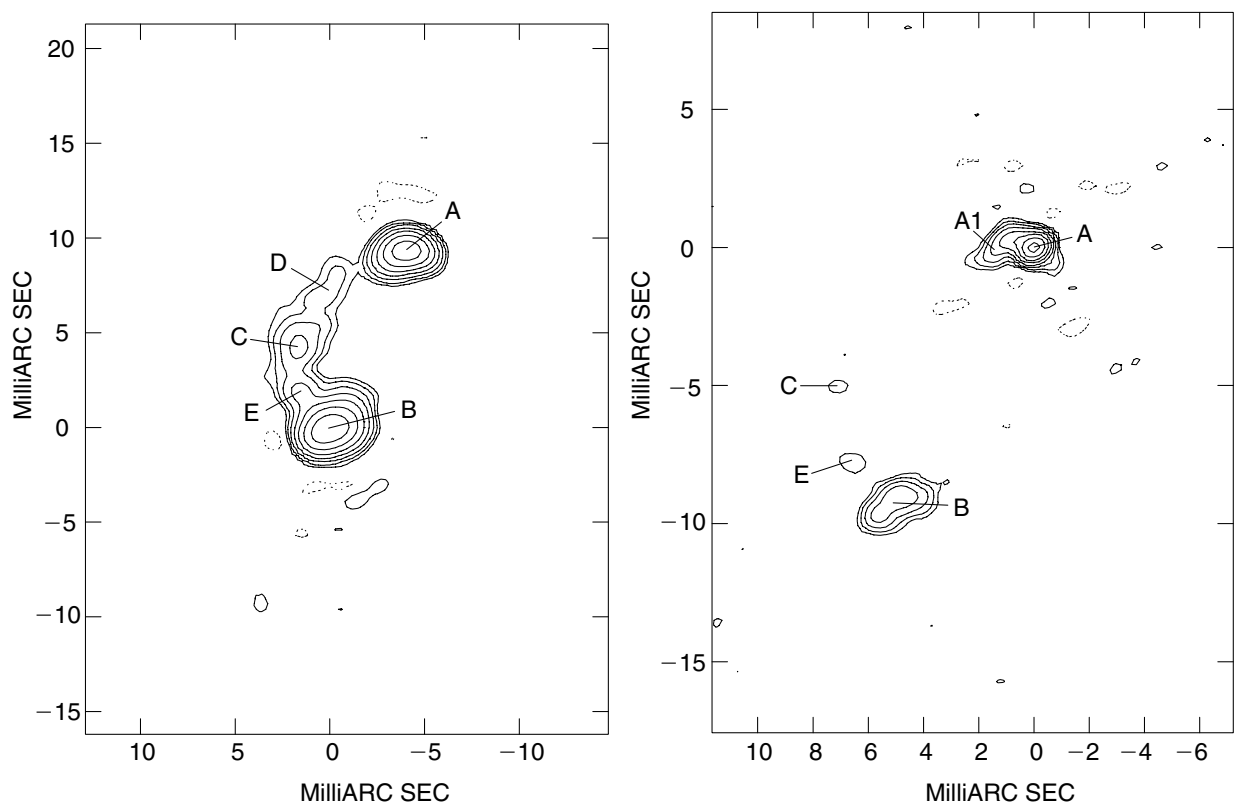

Figure 1 Left: $0153+744$ at $5 \mathrm{GHz}$. Peak flux $=237 \mathrm{mJy} / \mathrm{beam}$, contour levels $=-2,2,4,8,16,32,64,128,256 \mathrm{mJy} / \mathrm{beam}$. Right: $0153+744$ at $15 \mathrm{GHz}$. Peak flux $=168 \mathrm{mJy} / \mathrm{beam}$, contour levels $=-1,1,2,4,8,16,32,64,128,256 \mathrm{mJy} / \mathrm{beam}$.

Table 2. Observed source parameters derived from the images in Figures 1-3

\begin{tabular}{|c|c|c|c|c|c|c|c|c|c|c|c|c|}
\hline \multirow[t]{2}{*}{ Name } & \multirow[t]{2}{*}{$v \mathrm{GHz}$} & \multirow[t]{2}{*}{ Comp } & \multicolumn{3}{|c|}{ beam } & \multirow{2}{*}{$\begin{array}{l}\mathrm{rms} \\
\mathrm{mJy} / \mathrm{b}\end{array}$} & \multirow{2}{*}{$\begin{array}{l}\text { Maj } \\
\text { mas }\end{array}$} & \multirow{2}{*}{$\begin{array}{l}\text { Min } \\
\text { mas }\end{array}$} & \multirow{2}{*}{$\begin{array}{c}\mathrm{PA} \\
\circ\end{array}$} & \multirow{2}{*}{$\underset{\substack{\mathrm{p} \\
\mathrm{mJ} / \mathrm{b}}}{S}$} & \multirow{2}{*}{$\begin{array}{l}S_{\text {tot }} \\
\text { mJy }\end{array}$} & \multirow[t]{2}{*}{$\alpha$} \\
\hline & & & mas & mas & $\circ$ & & & & & & & \\
\hline \multirow[t]{5}{*}{$0153+744$} & 4.8 & A & 1.26 & 1.14 & 77.5 & 0.4 & 1.40 & 0.52 & 96.9 & 203.0 & 301.0 & 0.2 \\
\hline & & B & & & & & 1.83 & 1.02 & 113.1 & 237.0 & 503.0 & 2.0 \\
\hline & & $\mathrm{C}$ & & & & & 1.83 & 1.01 & 167.9 & 20.2 & 7.1 & 2.2 \\
\hline & & $\mathrm{D}$ & & & & & 4.32 & 0.78 & 143.6 & 6.3 & 5.7 & \\
\hline & & $\mathrm{E}$ & & & & & 1.89 & 0.68 & 37.0 & 22.7 & 14.6 & 2.1 \\
\hline \multirow[t]{5}{*}{$0153+744$} & 15 & A & 0.68 & 0.50 & -60.53 & 0.3 & 0.39 & 0.11 & 51.7 & 168.6 & 193.7 & \\
\hline & & A1 & & & & & 1.82 & 0.43 & 102.5 & 25.4 & 24.0 & \\
\hline & & B & & & & & 1.76 & 0.79 & 121.1 & 13.6 & 54.7 & \\
\hline & & $\mathrm{C}$ & & & & & - & - & - & 1.6 & 1.0 & \\
\hline & & $\mathrm{E}$ & & & & & 0.85 & 0.37 & 58.3 & 1.9 & 2.1 & \\
\hline $0133+476$ & 1.6 & & 6.54 & 3.90 & -0.77 & 1.4 & 3.52 & 2.12 & 158.9 & 944.5 & 1184.7 & \\
\hline $0133+476$ & 4.8 & & 1.97 & 1.43 & -13.99 & 0.9 & 0.80 & 0.20 & 158.8 & 1504.2 & 1627.1 & \\
\hline $0133+476$ & 8.4 & & 1.45 & 1.08 & 20.74 & 1.9 & 0.50 & 0.17 & 145.6 & 2332.4 & 2521.2 & -0 \\
\hline \multirow[t]{2}{*}{$0202+149$} & 8.4 & A & 1.98 & 1.12 & 26.3 & 1.8 & 0.50 & 0.18 & 145.5 & 1589.4 & 1825.9 & 0.8 \\
\hline & & B & & & & & - & - & - & 96.9 & 168.2 & 2.5 \\
\hline \multirow{2}{*}{$0202+149$} & 15 & A & 1.33 & 0.51 & -26.09 & 2.4 & 0.76 & 0.19 & 135.1 & 1079.6 & 1376.5 & \\
\hline & & B & & & & & 0.83 & 0.70 & 21.0 & 28.0 & 76.4 & \\
\hline
\end{tabular}

object was already known to have an inverted spectral index. We find that the radio spectrum peaks at a frequency $\geq 8.4 \mathrm{GHz}$, which makes it a new High Frequency Peaker source (Dallacasa et al. 2000; this workshop).

0202+149. This object has a 'core'-dominated structure (A) with a faint component (B) $\sim 7$ mas apart (Figure 3). The 'core' has spectral index $\alpha=0.8$, while component B has a steep spectral index $\alpha=2.5$ (Table 2). The radio spectrum peaks at around $400 \mathrm{MHz}$, which makes this a CSS source.

\section{Future Work}

The data for the following sources are still under analysis:

- $0859+470$ at $15 \mathrm{GHz}$;

- $0945+664$ at $1.6 \mathrm{GHz}, 5 \mathrm{GHz}, 15 \mathrm{GHz}$;

- $0954+556$ at $1.6 \mathrm{GHz}, 5 \mathrm{GHz}, 15 \mathrm{GHz}$.

The discovery of a few CSS/GPS sources of small size is important in order to finally have a complete flux limited sample of this class of objects (PW). These data will 

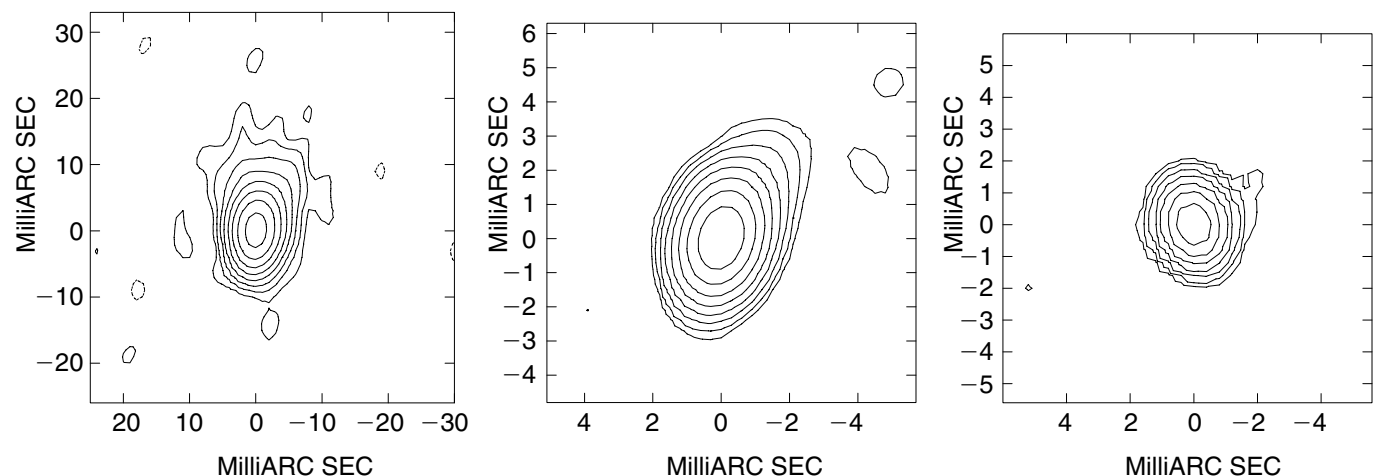

Figure 2 Left: $0133+476$ at $1.6 \mathrm{GHz}$. Peak flux $=944 \mathrm{mJy} / \mathrm{beam}$, contour levels $=-5,5,10,20,40,80,160,320,640,1200 \mathrm{mJy} / \mathrm{beam}$. Centre: $0133+476$ at $5 \mathrm{GHz}$. Peak flux $=1504 \mathrm{mJy} / \mathrm{beam}$, contour levels $=-7,7,14,28,56,112,224,448,856,1712 \mathrm{mJy} / \mathrm{beam}$. Right: $0133+476$ at $8.4 \mathrm{GHz}$. Peak flux $=2332 \mathrm{mJy} / \mathrm{beam}$, contour levels $=-20,20,40,80,160,320,640,1280,2560 \mathrm{mJy} / \mathrm{beam}$.
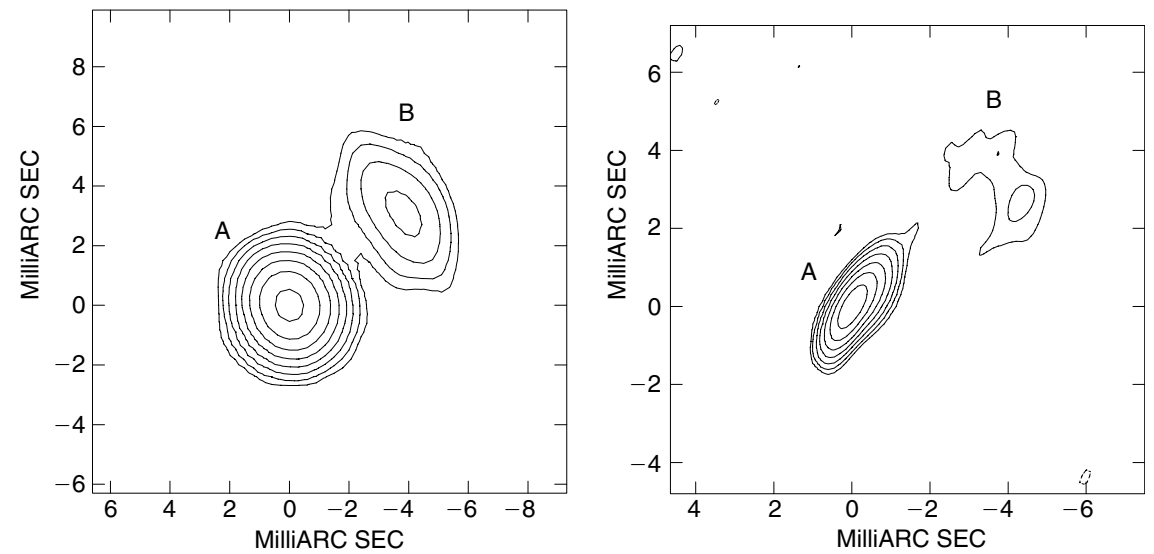

Figure 3 Left: $0202+149$ at $8.4 \mathrm{GHz}$. Peak flux $=1589 \mathrm{mJy} / \mathrm{beam}$. Right: $0202+149$ at $15 \mathrm{GHz}$. Peak flux $=1079.6 \mathrm{mJy} / \mathrm{beam}$. Contour levels $=-10,10,20,40,80,160,320,640,1280,3560 \mathrm{mJy} / \mathrm{beam}$.

complete the structure knowledge of the flat spectrum population of the PW sources. Combining the observations of bright CSS/GPS sources with our results will result in a complete flux limited sample spanning a range in source size of $\sim 10^{4}$, which is important to study source evolution.

\section{References}

Dallacasa, D., Stanghellini, C., Centonza, M., \& Fanti, R. 2000, A\&A, 363, 887

Hummel, C. A., Krichbaum, T. P., Witzel, A., Wuellner, K. H., Steffen, W., Alef, W., \& Fey, A. 1997, A\&A, 324, 857
Johnston, K. J., et al. 1995, AJ, 110, 880

Laing, R. A., Riley, J. M., \& Longair, M. S. 1983, MNRAS, 204, 151

O’Dea, C. 1998, PASP, 110, 493

O’Dea, C. P., Baum, S. A., \& Stanghellini, C. 1991, ApJ, 380, 660

Patnaik, A. R., Browne, I. W. A., Wilkinson, P. N., \& Wrobel, J. M. 1992, MNRAS, 254, 655P

Peacock, J. A., \& Wall, J. V. 1982, MNRAS, 198, 843 (PW)

Perlman, E. S., Padovani, P., Giommi, P., Sambruna, R., Jones, L. R., Tzioumis, A., \& Reynolds, J. 1998, AJ, 115, 1253P

Wright, A., \& Otrupcek, R. 1990, Parkes Catalogue, Australia Telescope National Facility 